\title{
Detection of Mediterranean Fruit Fly Larvae Ceratitis capitata (Diptera: Tephritidae) in Different Types of Fruit by HS-SPME GC-MS Method
}

\author{
Hasan Al-Khshemawee ${ }^{1,2}$, Manjree Agarwal ${ }^{1}, X_{i n}$ Du$^{1}$, Yonglin Ren ${ }^{*}$ \\ ${ }^{1}$ School of Veterinary and Life Science, Murdoch University, Murdoch, Australia \\ ${ }^{2}$ College of Agriculture, Wasit University, Wasit, Iraq \\ Email: *y.ren@murdoch.edu.au
}

How to cite this paper: Al-Khshemawee, H., Agarwal, M., Du, X. and Ren, Y.L. (2017) Detection of Mediterranean Fruit Fly Larvae Ceratitis capitata (Diptera: Tephritidae) in Different Types of Fruit by HS-SPME GC-MS Method. Journal of Biosciences and Medicines, 5, 154-169. https://doi.org/10.4236/jbm.2017.53017

Received: March 13, 2017

Accepted: March 31, 2017

Published: April 3, 2017

\begin{abstract}
Timely detection of Mediterranean fruit fly (Medfly) is very important so that eradication action can be taken on time. The larvae stage of this insect is the most dangerous stage as it is within the pulp of the fruit, making it hard to detect by visual inspection. In most countries at ports of entry the inspector check a small sample of fruit by visual inspection or by cutting the produce and searching for fungus and pests. This paper will investigate a quick, reliable and sensitive method to determine the presence of fruit flies. Our research focuses on developing the technology for detecting hidden infestations by using the Head Space-Soild Phase Micro Extraction (HS-SPME) method coupled with Gas Chromatography-Mass Spectrocopy (GC-MS) technique. Five different types of fruit were infested with an early stage of Medfly Ceratitis capitata Wiedemann (Diptera: Tephidae). We investigated to detect the differences in volatile organic compounds (VOC's) between infested and noninfested fruits by using HS-SPME with (GC-MS). The results indicated that for few chemicals no significant differences between infested and non-infested fruit can be seen, especially in the fruits with first instar. However, in case of third instar larvae infested fruits significant differences in the chemicals can be seen as compare to non infested fruits and other instar infestations. These chemicals include ethyl (Z)-2 butenoate, 2 -heptanone, anisole, $\beta$-cis-ocimene, $1,3,7$-nonatriene,4,8-dimethy-,ethyl octyate, isoamyl caproate and $1 \beta, 4 \beta \mathrm{h}, 10 \beta \mathrm{h}$ guaia-5,11-diene, in apple. Ethyl (Z)-2-butenoate, (+)-2-bornanone, (-)-transisopiperitenol, methyl caprate, caryophyllene and farnesene in orange. $\mathrm{Bu}-$ tanoic acid, 3-methyl-,2-methylbutul acetate, sabinene, $\beta$-myrcene, octanoic acid, methyl ester, dihydrocarvone, (-)-trans-isopiperitenol and ethyl laurate in mandarin. Butyl 2-methylbutanoate, terpinen-4-ol, P-menth-8-en-2-one,
\end{abstract}


E-,(3E,7E)-4,8,12-trimethyltrideca-1,3,7,11-tetraene and dodecanoic acid, ethyl ester in lemon. Decane, 3-methyl-, p-menth-1,4(8)-diene, 1-undecene and $\alpha$-cubebene in avocado. Thus, the VOC's method could provide a possible tool for detecting tephritid larvae and this method could be adopted by industries importing and exporting fruit.

\section{Keywords}

Ceratitis capitata, Infested Fruits, SPME-GC-MS, Volatile Compounds, Apple, Orange, Mandarin, Lemon, Avocado

\section{Introduction}

Mediterranean fruit fly Ceratitis capitata Wiedemann is species of invasive pest that affects fruit production and export worldwide. C. capitata attacks approximately 250 different species around the world [1]. United States spends about $\$ 57$ million per year on Medfly risk management [2]. Furthermore, over the period 2003-2008, Australian industry and government invested around \$128 million in the management of Medfly C. capitata [3]. Currently, fruit fly control is almost exclusively carried out with chemicals that are harmful to human health and to the environment [4]. In organic fruit production, the issue is more serious, since the law regarding organic farming prohibits the use of synthetic substances that include pesticides [5]. For this reason, farmers are trying to limit the problems by avoiding infection by Medfly. Results obtained in field and laboratory tests demonstrate different susceptibilities to Medfly damage [6]. Several types of research have demonstrated how different varieties of fruit display a variety of chemical profiles and how the release of their VOCs increases or decreases during the maturation process. Also, much attention has been a focused on the development of trapping systems for detection and monitoring of hidden infection [7]. However, improved methods are needed for detection of the immature stages as well, such as first, second and third instars. Larvae feed and develop while hidden within the pulp, making infestation difficult to detect by the senses, especially for Medfly eggs and first instar larvae which are clear to pale white in colour and hence camouflage well with fruit pulp and only $2-3 \mathrm{~mm}$ length inside the fruit [8]. In ports of entry, quarantine inspectors check import produce by taking small samples of fruit and checking for any signs of pests such as boring or feeding spots or by opening the fruit to search for Medfly larvae [9]. Research has shown that only $35 \%$ of fruit infested with fruit fly were detected by trained agricultural inspectors. If not checked by quarantine inspectors, infested fruits get distributed to consumers [8].

This paper will evaluate the use of Head Space-Solid Phase Micro Extraction (HS-SPME) method coupled with Gas Chromatography-Mass Spectroscopy (GC-MS) technique as a potential technology for improving detection of hidden insect infestation inside fruit. According to [10], medfly can change the volatile 
compounds of the fruits. It also has been shown that chemical changes can occur within host fruit as a result of insect infestation [11]. In this research, we examined different types of fruits infested with $C$. capitata to determine if infested fruit give us different chemicals profiles from non-infected fruits. Samples volatiles were collected at various stages of infections (first, second and third instars) of larvae, and chemical analysis was performed by GC-MS equipment.

\section{Material and Methods}

\subsection{Insects}

Medfly colony were obtained from the Department of Agriculture and Food, Western Australia (DAFWA) and reared in the Murdoch University Laboratory, in Perth Australia. All the flies were reared under conditions: $23^{\circ} \mathrm{C} \pm 2{ }^{\circ} \mathrm{C}$ and $75 \% \pm 5 \% \mathrm{RH}$, and 12:12-h (L:D) [12]. Adults are placed in screen cages $(40 \mathrm{~cm}$ length $\times 40 \mathrm{~cm}$ height $\times 40 \mathrm{~cm}$ depth) and each cage contained medfly food made from crystaline sugar from (Bidvest, Australia), yeast hydrolysate from (Australian Biosearch) in ratio of 4:1 and water $50 \mathrm{~mL}$. About 10-12 days after adult's emergence from pupae and mating of adults flies, eggs were collected every day, which are deposited on to mesh side and fallen into the water tray kept adjacent to the cage.

\subsection{Fruits}

Royal Gola Apple (Malus domestica) from New South Wales, Valencia orange (Citrus spp.) from Western Australia, Hass avocado (Persea Americana) from South-West of Western Australia, Hicksons Mandarin (Citrus reliculata) from New South Wales and Eureka Lemon (Citrus limon) from New South Wales were obtained from the local fruit and vegetable market. The fruits were stored for 2 days under $2^{\circ} \mathrm{C}$; twenty ripe fruits from each variety were used in our experiment.

\subsection{Infestation and Sample Preparation}

Fruits were cleaned with distilled water to remove any surface contaminants. Then, 30 eggs with $0.5 \mathrm{ml}$ of water were transferred to each single fruit by using a sterile syringe. Fruits were divided into three groups (5 fruits in each group) for volatiles analysis at different stages of larvae, first, second and third instars. Each group used two fruits to monitor the progress of larval development by cutting the fruit to determine the larvae stage and to evaluate the level of infestation by using a microscope to see the larvae stages and remaining three fruits were used for volatile analysis. In addition, one group was sampled as non-infested fruits. All the fruits were placed and stored in laboratory at a temperature of $24^{\circ} \mathrm{C}$ for 7 - 9 days; for the development of the larval stage. After collecting the volatile compounds as described in the next section, the various types of fruits were cut into small pieces and the number of larvae inside each fruit was counted. 


\subsection{Collection/Extraction of Volatile Compounds}

The analysis of compounds was focused on whole fruits. Various types of fruit were placed individually into 2 litre jars. One whole fruit was analysed in each jar. Volatiles were collected by solid phase micro extraction (SPME) fibre with 50/30 $\mu \mathrm{m}$ Carboxen/DVB/PDMS ( $2 \mathrm{~cm}$ ) (Sigma-Aldrich, Bellefonte, USA) coating. The samples were collected by inserting the fibre into the jar and exposing to the headspace. VOC's were collected on different times depending on the level of larvae inside the fruit. After sealing the jars for 16 hours at a temperature of $24^{\circ} \mathrm{C}$, the fibre was exposed to headspace for 2 hours which the optimized the HP-SPME extraction time. The desorption time of SPME fiber was $10 \mathrm{~min}$ in the injection port.

\subsection{Analysis of Volatile Compounds}

VOC's were analyzed with (Gas chromatography Agilent GCMS 7820A equipped with a mass spectrometer detector 5977E (Agilent Technologies, USA) and a DB-35ms column $(30 \mathrm{~m} \times 250 \mu \mathrm{m} \times 0.25 \mu \mathrm{m})$ (Santa Clara, CA 95051, USA). The carrier gas was $99.999 \%$ helium supplied by (BOC, gas, Sydney, Australia). The GC-MS operation conditions were as follows: The temperature of the injector port was $270^{\circ} \mathrm{C}$. The initial oven temperature was $50^{\circ} \mathrm{C}$ and increased to $250^{\circ} \mathrm{C}$ by $\left(5^{\circ} \mathrm{C} / \mathrm{min}\right)$. The column Flow rate was $1: 1 \mathrm{ml} / \mathrm{min}$ and splitless was 20 $\mathrm{ml} / \mathrm{min}$ at $1.5 \mathrm{~min}$. The total GCMS run time was $45 \mathrm{~min}$. Three experimental replicates were taken for each type of fruit. Compound peaks were deconvoluted by AMDIS version 2.72 and identified by searching the NIST 2014 MS database (the US National Institute of Standards and Technology) with retention index confirmation. Three replications for each type of fruit were analysed, and the experiment were repeated two times to confirm the chemicals.

\subsection{Method Sensitivity and Limit of Detection (LOD)}

The limit of detection was evaluated with alkane standard C7-C30 (Supelco, Bellefonte, USA). One litre Erlenmeyer flasks (Bibby Sterilin, Staffordshire, Cat. No. FE 1 L/3 equipped with cone/screw-thread adapter (Crown Scientific, Code ST 5313) with $1.1 \mathrm{~cm}$ blue septa (Grace Davison Discovery Sciences, catalog: 6518 ) were used make stock and diluted standard. The stock standard of concentration was prepared by adding $4 \mu$ of standard into sealed 1L Erlenmeyer flasks. Then, samples were diluted to ppb from ppm, ppt from ppb and ppq from ppt levels by transferring $1 \mathrm{~mL}$ of head space by syringe into another flask. After 1 hour of extraction time with 50/30 $\mu \mathrm{m}$ Carboxen/DVB/PDMS $(2 \mathrm{~cm}$ ) (SigmaAldrich, Bellefonte, USA) fibre at room temperature, the SPME fibre was injected into GC-MS with $270^{\circ} \mathrm{C}$ injection port. Each level was repeated two times.

\subsection{Statistical Analysis}

The number of larvae inside the fruit was analysed by one way (ANOVA). For the comparison of volatile compounds between different instars, the peak area was analysed by software using the two way (ANOVA) test [13]. Differences in 
the result were compared by using the least significant differences test (LSD $P \leq$ 0.05) for determining the means between different instars with non-infested fruits. The peak area was divided by $10^{6}$ for each single compound. The peaks left after subtracting from the blank run were only analysed.

\section{Results and Discussion}

\subsection{Level of Infestation}

The fruits were dissected immediately after collection of the volatiles compounds for finding out the level of infestation. For the first instar, it was hard to calculate the number of larvae; so the data was calculated by counting second instar larvae. The results indicated there were significant differences in the level of hatching in avocado compared to other types of fruit, like apple, lemon, orange and mandarin in laboratory conditions. Average \pm SD number of larvae per fruit was as follow: $8.06 \pm 1.58$ apple, $13.13 \pm 1.21$ orange, $11.93 \pm 1.77$ mandarin, $9.06 \pm$ 1.81 lemon and $18.93 \pm 1.10$ avocado (Figure 1). The non-infested fruit was also dissected to confirm there are no natural infestations by Medfly.

\subsection{Limit of Detection (LOD)}

The GC-MS response of the stock and diluted alkanes standard decreased form ppm (Parts per million) level to ppt (parts per trillion) level (Table 1). Some of the alkanes can be detected less than ppt level with SPME method. Octane, decane, undecane, pentadecane, hexadecane and heptadecane can be detected in ppt level. Octane can be detected in small amount even at ppq level.

Table 1. Limits of detection (LOD) of C7-C30 standard at three levels by using 50/30 $\mu \mathrm{m}$ Carboxen/DVB/PDMS fibre.

\begin{tabular}{|c|c|c|c|c|c|c|}
\hline Standard & Formula & $\mathrm{RI}^{\mathrm{a}}$ & LOD $(\mathrm{ppm})^{\mathrm{b}}$ & LOD $(p p b)^{c}$ & LOD $(p p t)^{\mathrm{d}}$ & Linearity $\left(r^{2}\right)^{e}$ \\
\hline Octane & C8H18 & 729.1 & 100.061 & 33.394 & 5.050 & 0.948 \\
\hline Nonane & С9H20 & 899.9 & 141.900 & 57.492 & n.d & 0.988 \\
\hline Decane & $\mathrm{C} 10 \mathrm{H} 22$ & 1000.8 & 224.999 & 74.246 & 4.395 & 0.957 \\
\hline Undecane & $\mathrm{C} 11 \mathrm{H} 24$ & 1100.6 & 164.794 & 77.475 & 2.983 & 0.997 \\
\hline Dodecane & $\mathrm{C} 12 \mathrm{H} 26$ & 1200.8 & 24.028 & 6.819 & n.d & 0.941 \\
\hline Tridecane & $\mathrm{C} 13 \mathrm{H} 28$ & 1300.6 & 56.533 & 27.538 & n.d & 0.998 \\
\hline Tetradecane & $\mathrm{C} 14 \mathrm{H} 30$ & 1399 & 22.945 & 7.431 & n.d & 0.960 \\
\hline Pentadecane & $\mathrm{C} 15 \mathrm{H} 32$ & 1500.5 & 17.307 & 10.262 & 1.068 & 0.994 \\
\hline Hexadecane & $\mathrm{C} 16 \mathrm{H} 34$ & 1600.6 & 6.798 & 3.440 & 2.070 & 0.944 \\
\hline Heptadecane & C17H36 & 1700.5 & 6.499 & 2.521 & 0.861 & 0.946 \\
\hline Nodaecane & $\mathrm{C} 19 \mathrm{H} 40$ & 1900 & 13.734 & n.d & n.d & - \\
\hline Eicosane & $\mathrm{C} 2 \mathrm{OH} 42$ & 2000.8 & 15.983 & 4.987 & n.d & 0.955 \\
\hline Heneicosane & $\mathrm{C} 21 \mathrm{H} 44$ & 2100 & 18.651 & n.d & n.d & - \\
\hline Tricosane & $\mathrm{C} 23 \mathrm{H} 48$ & 2299.5 & 20.503 & n.d & n.d & - \\
\hline Tetracosane & $\mathrm{C} 24 \mathrm{H} 50$ & 2400.8 & 15.119 & n.d & n.d & - \\
\hline Pentacosane & $\mathrm{C} 25 \mathrm{H} 52$ & 2499 & 10.042 & n.d & n.d & - \\
\hline
\end{tabular}

$\mathrm{a}=$ retention index; $\mathrm{b}=$ parts per million; $\mathrm{c}=$ parts per billion; $\mathrm{d}=$ parts per trillion; $\mathrm{e}=$ Regression coefficient. 


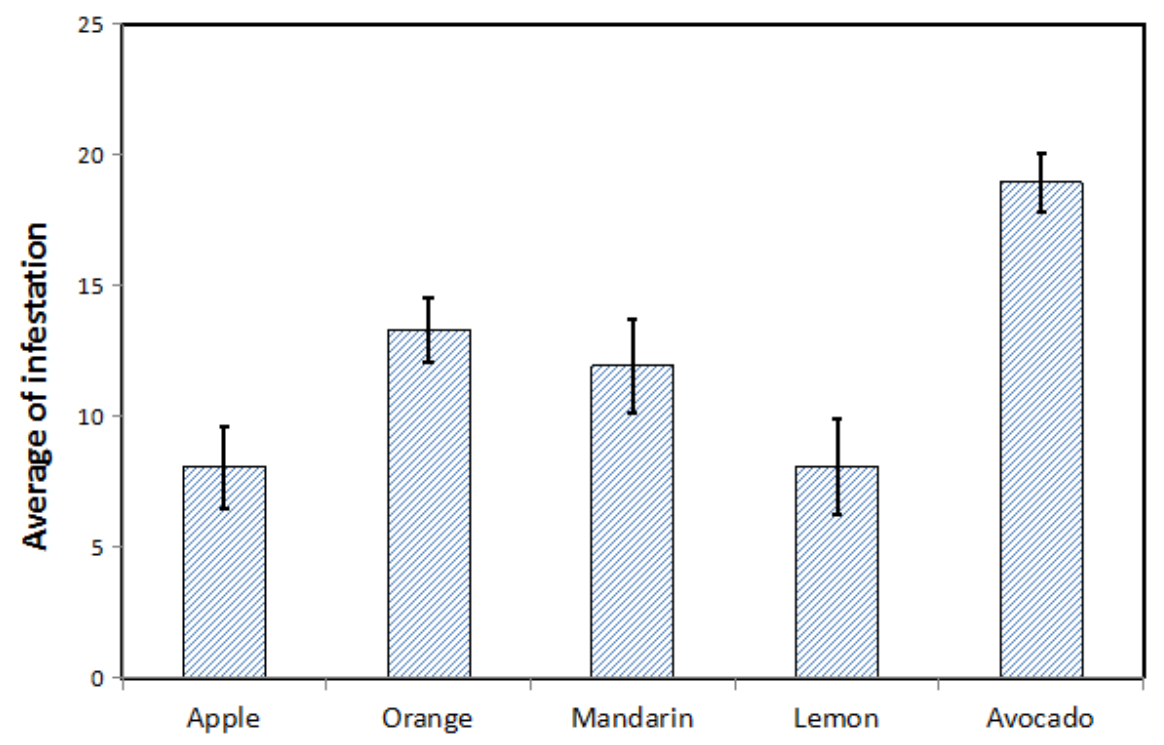

Figure 1. Infestation level (Mean $\pm \mathrm{SD}$ ) in different fruits by $C$. capitata.

\subsection{The Volatiles Compounds from Fruit}

There were many differences between compounds for each type of fruits and also between infested and non infested fruits. Some of the compounds were detected in one type of fruit, was found to be absent in another type. From the GC analysis, about 33 compounds from apple, 45 compounds from orange, 45 compounds from mandarin, 44 compounds from lemon, and 40 compounds from avocado were identified. All these compounds were identified by comparing with the retention index in the literature (NIST) and mass spectra in the NIST. We analysed 23 compounds each from apple (Table 2), orange (Table 3), mandarin (Table 4), and lemon (Table 5), while 17 compounds were analysed from avocado (Table 6). The comparison of main compounds between different types of fruit infested with three different instars is explained in Figure 2.

\subsubsection{The Volatile Compounds from Apples}

In case of non infested apples, the main peaks were hexyl acetate, n-butyl 2 methylbutyrate, $n$-hexyl propionate and isobutyl caproate. Many compounds were detected in fruits infested with third instar larvae but not in fruits infested with first or second instar larvae or in non infested fruit and these included ethyl (Z)-2 butenoate, 2 -heptanone, anisole, $\beta$-cis-ocimene, 1,3,7-nonatriene,4,8-dimethy-, ethyl octyate, isoamyl caproate, ethyl decylate and $1 \beta, 4 \beta \mathrm{h}, 10 \beta \mathrm{h}$-guaia-5,11-diene (Table 2). The most significant compounds comparing with non infested fruits in third instar were propyl isobutyrate, 1-hexanol, hexyl acetate, n-butyl 2 methylbutyrate, $n$-hexyl propionate and isobutyl caproate. In the second instar, they were propyl isobutyrate, 1-hexanol, hexyl acetate, n-butyl 2 methylbutyrate and $n$-hexyl propionate, while the significant chemicals which were recorded in first instar were propyl isobutyrate, hexyl acetate and isobutyl caproate (Table 2). The concentration of 1-hexanol and styrene increased with increase in instars, however, propyl isobutyrate, hexyl acetate, n-butyl 2 methylbutyrate and 
Table 2. GC peak area (one unit corresponds to a $10^{6}$ area) of volatile compounds in apple infested with $C$. capitata detected by GC-MS.

\begin{tabular}{|c|c|c|c|c|c|c|c|c|}
\hline \multirow{2}{*}{ Compounds } & \multirow{2}{*}{ RT } & \multirow{2}{*}{ RI } & \multirow{2}{*}{ RIL } & \multirow{2}{*}{ Prob.\% } & \multicolumn{3}{|c|}{ Infested } & \multirow{2}{*}{ Non-infested } \\
\hline & & & & & 1 instar & 2 instar & 3 instar & \\
\hline Ethyl(Z)-2 butenoate & 5.45 & 848.9 & 830 & 89 & n.d. & n.d. & 0.77 & n.d. \\
\hline Propyl isobutyrate & 5.85 & 856.8 & 861 & 86 & $9.35^{*}$ & $3.89^{*}$ & $2.23^{*}$ & 24.07 \\
\hline 1-Hexanol & 6.26 & 872.3 & 860 & 89 & $35.51^{\mathrm{ns}}$ & $50.69^{*}$ & $78.72^{*}$ & 10.58 \\
\hline Styrene & 6.95 & 801.7 & 883 & 87 & 0.24 & 0.12 & 2.04 & n.d. \\
\hline 2-Heptanone & 6.97 & 891.7 & 871 & 83 & n.d. & n.d. & 17.72 & n.d. \\
\hline Anisole & 7.91 & 916.9 & 898 & 92 & n.d. & n.d. & 0.55 & n.d. \\
\hline Benzaldehyde & 9.44 & 895.7 & 982 & 79 & 0.33 & n.d. & 1.18 & n.d. \\
\hline Hexyl acetate & 11.42 & 973.7 & 984 & 88 & $419.33^{*}$ & $411.08^{\star}$ & $396.33^{*}$ & 544.26 \\
\hline n-Butyl 2 methylbutyrate & 12.41 & 1044.9 & 1019 & 84 & $210.68^{\text {ns }}$ & $88.18^{*}$ & $75.64^{*}$ & 215.87 \\
\hline$\beta$-cis-Ocimene & 12.60 & 1049.9 & 1041 & 89 & n.d. & n.d. & 0.46 & n.d. \\
\hline 2-Methylbutyl 2-methylbutyrate & 14.45 & 1105.3 & 1090 & 80 & 14.53 & n.d. & 19.05 & n.d. \\
\hline n-Hexyl propionate & 14.56 & 1085.3 & 1083 & 94 & $151.50^{\mathrm{ns}}$ & $81.41^{\star}$ & $80.19^{*}$ & 139.94 \\
\hline 1,3,7-Nonatriene,4,8-dimethy-, & 14.84 & 1108.3 & 1089 & 83 & n.d. & n.d. & 21.08 & n.d. \\
\hline Methyl caprylate & 15.11 & 1126.5 & 1108 & 76 & 0.6 & 0.35 & 5.23 & n.d. \\
\hline Dodecane & 17.40 & 1201.7 & 1200 & 81 & 1.19 & 5.22 & n.d. & n.d. \\
\hline Ethyl octyate & 17.39 & 1199.8 & 1175 & 95 & n.d. & n.d. & 48.4 & n.d. \\
\hline Decanal & 17.61 & 1199.6 & 1204 & 85 & 1.19 & 1.01 & 1.20 & n.d. \\
\hline Isoamyl caproate & 18.90 & 1252.4 & 1253 & 90 & n.d. & n.d. & 1.76 & n.d. \\
\hline Ethyl decylate & 22.86 & 1390.4 & 1381 & 88 & n.d. & n.d. & 1.07 & n.d. \\
\hline $1 \beta, 4 \beta \mathrm{h}, 10 \beta \mathrm{h}$-Guaia-5,11-diene & 24.93 & 1462.1 & 1469 & 93 & n.d. & n.d. & 0.85 & n.d. \\
\hline
\end{tabular}

${ }^{*}$ Means there are significant differences between infested fruit and non-infested fruit (LSD mean $\mathrm{P} \leq 0.05$ ). Prob\% means percent of probability. ns means there are no significant differences between infested fruit and non-infested fruit (LSD mean $\mathrm{P} \leq 0.05$ ). n.d. means compounds are not detected. (RT) retention time, (RI) retention index, (RIL) Literature retention index (NIST).

Table 3. GC peak area (one unit corresponds to a $10^{6}$ area) of volatile compounds in orange infested with $C$. capitata detected by GC-MS.

\begin{tabular}{|c|c|c|c|c|c|c|c|c|}
\hline \multirow{2}{*}{ Compounds } & \multirow{2}{*}{ RT } & \multirow{2}{*}{ RI } & \multirow{2}{*}{ RIL } & \multirow{2}{*}{ Prob. $\%$} & \multicolumn{3}{|c|}{ Infested } & \multirow{2}{*}{ Non-infested } \\
\hline & & & & & 1 instar & 2 instar & 3 instar & \\
\hline Ethyl (Z)-2-butenoate & 5.45 & 849.6 & 830 & 89 & n.d. & n.d. & 2.04 & n.d. \\
\hline$\beta$-phellandrene & 8.26 & 850.2 & 964 & 81 & 0.95 & n.d. & 11.38 & n.d. \\
\hline n-Butyl butyrate & 9.28 & 957.6 & 939 & 74 & $29.13^{\text {ns }}$ & $57.79^{\text {ns }}$ & n.d. & 32.76 \\
\hline Myrcene & 10.63 & 992.5 & 979 & 89 & $19.16^{\mathrm{ns}}$ & $40.7^{\mathrm{ns}}$ & $242.02^{*}$ & 12.58 \\
\hline 3-Carene & 11.21 & 1010.7 & 1005 & 89 & $124.85^{\star}$ & $252.90^{\star}$ & $115.88^{\star}$ & 22.92 \\
\hline D-Limonene & 11.88 & 1031.4 & 1018 & 86 & $389.93^{*}$ & $614.20^{*}$ & $799.27^{\star}$ & 49.73 \\
\hline$\beta$-cis-Ocimene & 12.60 & 1049.9 & 1041 & 81 & 1.22 & 5.34 & 24.95 & n.d. \\
\hline Methyl caprylate & 15.11 & 1124.2 & 1134 & 91 & $4.35^{\mathrm{ns}}$ & $244.38^{*}$ & $123.45^{\star}$ & 2.23 \\
\hline$(+)$-2-Bornanone & 15.67 & 1146.7 & 1141 & 87 & n.d. & n.d. & 0.76 & n.d. \\
\hline Isobutyl caproate & 15.94 & 1137.3 & 1118 & 78 & $348.61^{\mathrm{ns}}$ & $313.61^{*}$ & $144.03^{*}$ & 391.66 \\
\hline
\end{tabular}




\section{Continued}

\begin{tabular}{|c|c|c|c|c|c|c|c|c|}
\hline Ethyl octyate & 17.40 & 1153.8 & 1152 & 96 & $155.85^{*}$ & $188.26^{*}$ & $208.95^{*}$ & 49.67 \\
\hline (-)-trans-Isopiperitenol & 17.57 & 1206.0 & 1206 & 82 & n.d. & n.d. & 15.50 & n.d. \\
\hline Butyl(2E)-2-hexenoate & 18.63 & 1243.1 & 1243 & 92 & n.d. & 50.13 & 22.16 & n.d. \\
\hline Isoamyl caproate & 18.90 & 1252.6 & 1253 & 82 & 7.49 & 40.48 & 37.81 & n.d. \\
\hline Methyl caprate & 20.96 & 1324.4 & 1309 & 80 & n.d. & n.d. & 0.90 & n.d. \\
\hline limonene-1,2-dial & 21.38 & 1340.8 & 1342 & 84 & 4.59 & 13.56 & 57.27 & n.d. \\
\hline Octanoic acid n-butyl ester & 21.58 & 1346.5 & 1348 & 87 & n.d. & 66.55 & 3.22 & n.d. \\
\hline Eugenol & 21.88 & 1356.0 & 1337 & 90 & n.d. & 0.25 & 9.79 & n.d. \\
\hline Caryophyllene & 23.54 & 1414.4 & 1424 & 87 & n.d. & n.d. & 52.50 & n.d. \\
\hline Valencen & 25.38 & 1478.2 & 1474 & 86 & $885.93^{\text {ns }}$ & $755.80^{*}$ & $636.74^{*}$ & 903.59 \\
\hline Farnesene & 25.70 & 1488.9 & 1499 & 84 & n.d. & n.d. & 42.70 & n.d. \\
\hline
\end{tabular}

${ }^{*}$ Means there are significant differences between infested fruit and non-infested fruit (LSD mean $\mathrm{P} \leq 0.05$ ). Prob\% means percent of probability. ns means there are no major differences between infested fruit and non-infested fruit (LSD mean $\mathrm{P} \leq 0.05$ ). n.d. means compounds are not detected. (RT) retention time, (RI) retention index, (RIL) Literature retention index (NIST).

Table 4. GC peak area (one unit corresponds to a $10^{6}$ area) of volatile compounds in mandarin infested with C. capitata detected by GC-MS.

\begin{tabular}{|c|c|c|c|c|c|c|c|c|}
\hline \multirow{2}{*}{ Compounds } & \multirow{2}{*}{$\mathrm{RT}$} & \multirow{2}{*}{ RI } & \multirow{2}{*}{ RIL } & \multirow{2}{*}{ Prob.\% } & \multicolumn{3}{|c|}{ Infested } & \multirow{2}{*}{ Non-infested } \\
\hline & & & & & 1 instar & 2 instar & 3 instar & \\
\hline Butanoic acid, 3-methyl- & 5.42 & 849.8 & 830 & 88 & n.d. & n.d. & 2.86 & n.d. \\
\hline 2-methylbutul acetate & 6.64 & 882.0 & 868 & 81 & n.d. & n.d. & 1.2 & n.d. \\
\hline 1R-a-Pinene & 8.47 & 932.8 & 922 & 85 & 1.16 & 5.87 & n.d. & n.d. \\
\hline Mesitylene & 9.74 & 968.0 & 956 & 82 & $0.71^{\mathrm{ns}}$ & n.d. & n.d. & 4.26 \\
\hline Sabinene & 9.96 & 973.9 & 975 & 89 & n.d. & n.d. & 129.64 & n.d. \\
\hline Terebenthene & 10.00 & 970.0 & 975 & 87 & n.d. & 49.52 & 168.13 & n.d. \\
\hline$\beta$-Myrcene & 10.63 & 992.5 & 979 & 89 & n.d. & n.d. & 86.54 & n.d. \\
\hline 3-Carene & 11.21 & 1010.7 & 1005 & 89 & $3.24^{\mathrm{ns}}$ & $25.88^{\star}$ & $25.99^{*}$ & 0.24 \\
\hline D-Limonene & 11.88 & 1031.4 & 1018 & 89 & $625.23^{*}$ & $751.71^{\star}$ & $907.88^{*}$ & 518.48 \\
\hline Moslene & 12.90 & 1059.5 & 1047 & 85 & $5.08^{*}$ & $17.68^{\text {ns }}$ & $57.21^{\star}$ & 22.13 \\
\hline p-menth-1,4(8)-diene & 13.64 & 1088.3 & 1080 & 83 & 3.27 & 9.92 & 32.33 & n.d. \\
\hline Octanoic acid, methyl ester & 15.14 & 1126.5 & 1109 & 84 & n.d. & n.d. & 3.75 & n.d. \\
\hline (-)-Terpinen-4-ol & 16.74 & 1178.9 & 1161 & 84 & n.d. & 6.62 & 51.19 & n.d. \\
\hline$\alpha$-Terpineol & 17.11 & 1192.5 & 1172 & 88 & n.d. & 3.22 & 20.46 & n.d. \\
\hline Dihydrocarvone & 17.37 & 1197.4 & 1189 & 80 & n.d. & n.d. & 43.36 & n.d. \\
\hline Dodecane & 17.40 & 1200.1 & 1200 & 89 & $4.03^{\mathrm{ns}}$ & n.d. & n.d. & 4.62 \\
\hline (-)-trans-Isopiperitenol & 17.44 & 1206.0 & 1206 & 85 & n.d. & n.d. & 8.03 & n.d. \\
\hline p-Mentha-1(7),8(10)-dien-9-ol & 20.03 & 1340.5 & 1340 & 82 & n.d. & n.d. & 2.64 & n.d. \\
\hline Tridecane & 20.28 & 1299.9 & 1300 & 81 & $3.59^{\mathrm{ns}}$ & $2.79^{\text {ns }}$ & n.d. & 4.01 \\
\hline 1,2-Cyclohexanediol, 1-methyl-4-(1- & 21.37 & 1338.8 & 1342 & 96 & n.d. & 3.25 & 19.49 & n.d. \\
\hline$(-)$ - $\beta$-Elmene & 22.80 & 1388.1 & 1387 & 83 & n.d. & 2.55 & 3.97 & n.d. \\
\hline (+)-epi-Bicyclosesquiphellandrene & 23.79 & 1421.7 & 1428 & 81 & 0.41 & 0.87 & 1.98 & n.d. \\
\hline Ethyl laurate & 27.73 & 1598.7 & 1580 & 90 & n.d. & n.d. & 1.23 & n.d. \\
\hline
\end{tabular}

${ }^{*}$ Means there are significant differences between infested fruit and non-infested fruit (LSD mean $\left.\mathrm{P} \leq 0.05\right)$. Prob\% means percent of probability. ns means there are no significant differences between infested fruit and non-infested fruit (LSD mean $\mathrm{P} \leq 0.05)$. n.d. means compounds are not detected. (RT) retention time, (RI) retention index, (RIL) Literature retention index (NIST). 
Table 5. GC peak area (one unit corresponds to a $10^{6}$ area) of volatile compounds in lemon infested with $C$. capitata detected by GC-MS.

\begin{tabular}{|c|c|c|c|c|c|c|c|c|}
\hline \multirow{2}{*}{ Compounds } & \multirow{2}{*}{ RT } & \multirow{2}{*}{ RI } & \multirow{2}{*}{ RIL } & \multirow{2}{*}{ Prob.\% } & \multicolumn{3}{|c|}{ Infested } & \multirow{2}{*}{ Non-infested } \\
\hline & & & & & 1 instar & 2 instar & 3 instar & \\
\hline Sabinene & 9.96 & 973.2 & 975 & 89 & $21.21^{\mathrm{ns}}$ & 238.72 & 399.21 & n.d. \\
\hline D-Limonene & 11.88 & 1031.4 & 1018 & 89 & $352.62^{*}$ & $625.74^{*}$ & $767.11^{\star}$ & 95.4 \\
\hline Butyl 2-methylbutanoate & 12.40 & 1044.8 & 1026 & 85 & n.d. & n.d. & 0.99 & n.d. \\
\hline Benzene,1-methyl-3-(1-methylethenyl)- & 13.91 & 1089.2 & 1099 & 84 & $6.26^{\mathrm{ns}}$ & n.d. & n.d. & 10.88 \\
\hline (E)4,8-Dimethyl-1,3,7-Noatriene & 14.88 & 1119.0 & 1116 & 86 & 277.41 & 136.05 & 183.96 & n.d. \\
\hline Methyl caprylate & 15.11 & 1126.5 & 1109 & 86 & $3.87^{\mathrm{ns}}$ & $55.20^{\star}$ & $317.42^{*}$ & 2.81 \\
\hline Limonene oxide, trans- & 15.51 & 1139.4 & 1130 & 88 & $7.88^{\mathrm{ns}}$ & n.d. & n.d. & 10.09 \\
\hline$(+)$-2-Bornanone & 15.67 & 1146.7 & 1141 & 86 & n.d. & 8.11 & 18.83 & n.d. \\
\hline Terpinen-4-ol & 16.79 & 1178.9 & 1161 & 84 & n.d. & n.d. & 86.01 & n.d. \\
\hline a-Terpineol & 17.11 & 1192.5 & 1172 & 83 & 5.97 & 36.04 & 179.05 & n.d. \\
\hline P-Menth-8-en-2-one,E- & 17.57 & 1088.3 & 1080 & 82 & n.d. & n.d. & 5.79 & n.d. \\
\hline Hexyl 2-methylbutyrate & 18.51 & 1238.6 & 1232 & 90 & $29.81^{*}$ & $14.20^{\text {ns }}$ & $46.60^{*}$ & 11.84 \\
\hline Tridecane & 20.28 & 1299.9 & 1300 & 81 & $2.13^{\text {ns }}$ & n.d. & n.d. & 3.15 \\
\hline Decanoic acid, methyl ester & 20.96 & 1324.1 & 1309 & 81 & n.d. & 13.41 & 31.97 & n.d. \\
\hline Limonene-1,2-diol & 21.37 & 1340.8 & 1342 & 93 & $4.16^{\mathrm{ns}}$ & $42.17^{*}$ & $7.96^{\mathrm{ns}}$ & 4.4 \\
\hline$(-)-\beta$-Elmene & 22.80 & 1388.1 & 1387 & 88 & $122.59^{*}$ & $106.96^{\mathrm{ns}}$ & $35.50^{\text {ns }}$ & 77.31 \\
\hline 7-epi-a-selinene & 26.05 & 1501.0 & 1503 & 85 & 87.3 & 190.56 & n.d. & n.d. \\
\hline E-Nerolidol & 27.02 & 1534.9 & 1548 & 85 & $65.53^{*}$ & $31.74^{\mathrm{ns}}$ & $47.65^{\mathrm{ns}}$ & 0.87 \\
\hline (3E,7E)-4,8,12-Trimethyltrideca-1,3,7,11-tetraene & 27.38 & 1547.3 & 1557 & 55 & n.d. & n.d & 1.98 & n.d. \\
\hline Dodecanoic acid, ethyl ester & 27.72 & 1559.2 & 1566 & 87 & n.d. & n.d. & 0.8 & n.d. \\
\hline Intermedol & 29.20 & 1610.9 & 1630 & 81 & n.d. & $7.52^{\mathrm{ns}}$ & $122.58^{\star}$ & 4.23 \\
\hline
\end{tabular}

${ }^{*}$ Means there are significant differences between infested fruit and non-infested fruit (LSD mean $\left.\mathrm{P} \leq 0.05\right)$. Prob\% means percent of probability. ns means there are no significant differences between infested fruit and non-infested fruit (LSD mean $\mathrm{P} \leq 0.05)$. n.d. means compounds are not detected. (RT) retention time, (RI) retention index, (RIL) Literature retention index (NIST).

Table 6. GC peak area (one unit corresponds to a $10^{6}$ area) of volatile compounds in avocado infested with $C$. capitata detected by GC-MS.

\begin{tabular}{|c|c|c|c|c|c|c|c|c|}
\hline \multirow{2}{*}{ Compounds } & \multirow{2}{*}{ RT } & \multirow{2}{*}{ RI } & \multirow{2}{*}{ RIL } & \multirow{2}{*}{ Prob.\% } & \multicolumn{3}{|c|}{ Infested } & \multirow{2}{*}{ Non-infested } \\
\hline & & & & & 1 instar & 2 instar & 3 instar & \\
\hline 1-Heptanal & 7.36 & 898.8 & 882 & 88 & $1.04^{\mathrm{ns}}$ & $0.81^{\mathrm{ns}}$ & n.d. & 0.44 \\
\hline Sulcatone & 10.48 & 973.9 & 964 & 80 & $0.58^{\mathrm{ns}}$ & $33.85^{*}$ & $1.35^{\mathrm{ns}}$ & 0.53 \\
\hline Hexyl acetate & 11.42 & 973.7 & 984 & 84 & $0.51^{\mathrm{ns}}$ & n.d. & n.d. & 0.23 \\
\hline D-Limonene & 11.88 & 1031.4 & 1018 & 90 & $23.56^{*}$ & $114.96^{*}$ & $131.75^{\star}$ & 94.70 \\
\hline Moslene & 12.90 & 1058.3 & 1047 & 86 & $0.75^{*}$ & $51.88^{*}$ & $2.87^{\mathrm{ns}}$ & 7.19 \\
\hline
\end{tabular}




\begin{tabular}{|c|c|c|c|c|c|c|c|c|}
\hline Decane, 3-methyl- & 13.31 & 1070.5 & 1072 & 82 & n.d. & n.d. & 0.22 & n.d. \\
\hline p-menth-1,4(8)-diene & 13.88 & 1088.3 & 1080 & 81 & n.d. & n.d. & $0.38^{\text {ns }}$ & 1.49 \\
\hline 1-undecene & 14.00 & 1091.7 & 1088 & 79 & n.d. & n.d. & 0.13 & n.d. \\
\hline E-4,8-Dimethyl-1,3,7-Noatriene & 14.88 & 1119.0 & 1116 & 86 & $16.19^{\text {ns }}$ & $14.31^{*}$ & $2.02^{\star}$ & 11.69 \\
\hline$\alpha$-Terpineol & 17.11 & 1192.5 & 1172 & 81 & n.d. & 6.41 & 0.19 & n.d. \\
\hline 1,2,6-Dimethylundecane & 17.81 & 1214.6 & 1216 & 81 & 0.45 & 1.21 & 0.55 & n.d. \\
\hline Limonene glycol & 21.38 & 1338.2 & 1342 & 83 & n.d. & 2.69 & n.d. & n.d. \\
\hline a-Cubebene & 21.66 & 1348.4 & 1350 & 82 & n.d. & n.d. & 0.22 & n.d. \\
\hline Meraneine & 22.03 & 1361.3 & 1342 & 85 & n.d. & 0.41 & n.d. & n.d. \\
\hline $\begin{array}{l}\text { (4R,4aS,6S)-4,4a-Dimethyl-6-(prop-1-en-2-yl)- } \\
\text { 1,2,3,4,4a,5,6,7-octahydronaphthalene }\end{array}$ & 24.79 & 1457.4 & 1475 & 82 & $0.32^{\mathrm{ns}}$ & $10.64^{\star}$ & $2.54^{\mathrm{ns}}$ & 0.61 \\
\hline $1 \beta, 4 \beta \mathrm{h}, 10 \beta \mathrm{h}$-Guaia-5,11-diene & 24.93 & 1462.0 & 1469 & 83 & $1.55^{\mathrm{ns}}$ & n.d. & n.d. & 1.64 \\
\hline Valencen & 25.38 & 1479.9 & 1492 & 89 & $61.82^{\mathrm{ns}}$ & $3.31^{*}$ & $14.60^{*}$ & 61.13 \\
\hline
\end{tabular}

${ }^{*}$ Means there are significant differences between infested fruit and non-infested fruit (LSD mean $\mathrm{P} \leq 0.05$ ). Prob\% means percent of probability. ns means there are no significant differences between infested fruit and non-infested fruit (LSD mean P $\leq 0.05$ ). n.d. means compounds are not detected. (RT) retention time, (RI) retention index, (RIL) Literature retention index (NIST).
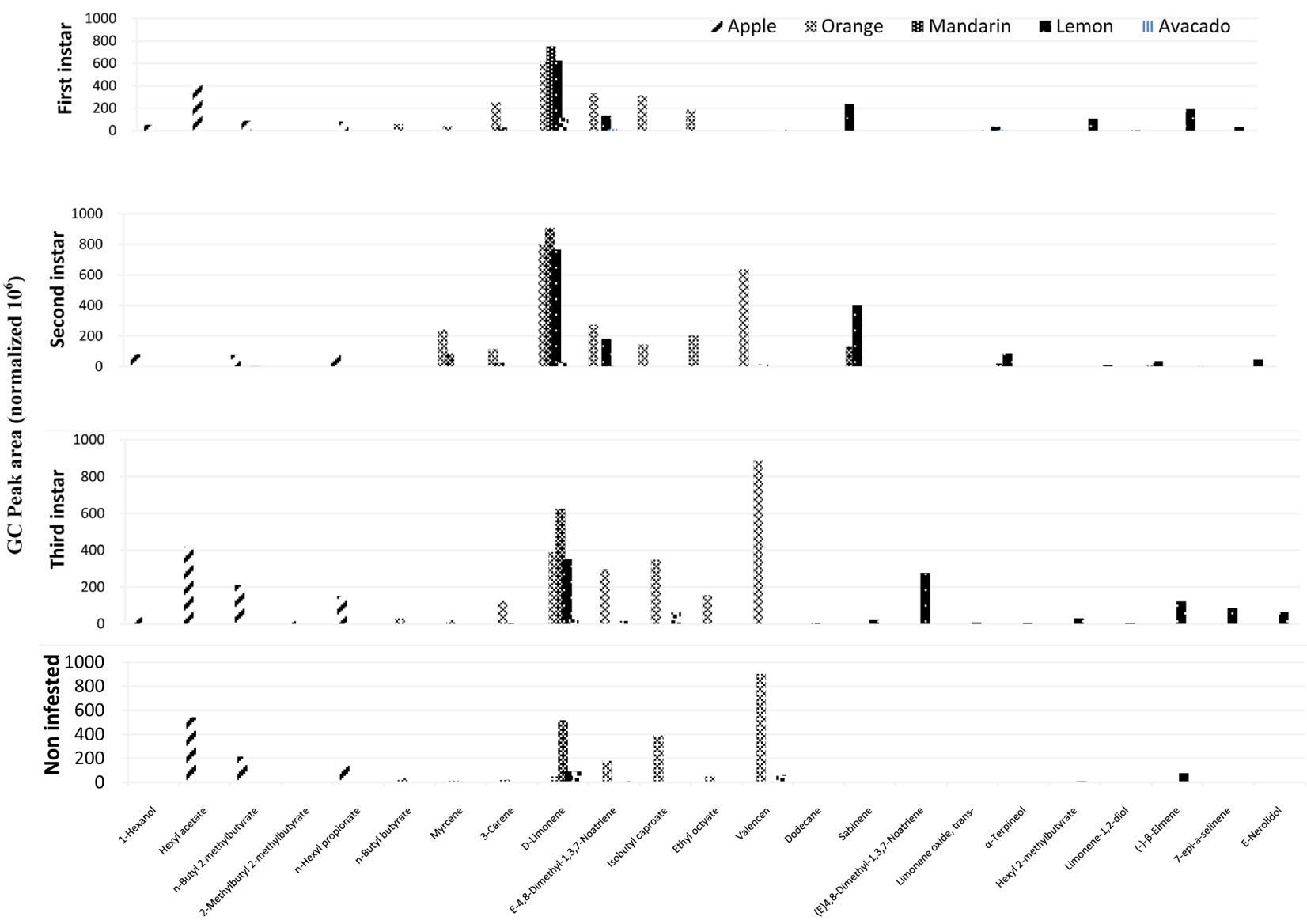

Figure 2. Comparison of main peaks between different types of fruits at three different instars. 
$\mathrm{n}$-hexyl propionate were decreased with increase in instars. From these results, it shows that the level of larvae can change the profile of compounds as also reported by [14]. Also, these compounds may impact on medfly larvae behaviour [15]. Infested apple with codling moth (Cydia pomonella L.) larvae gave a high level of esters and farnescene, from first instar infested and other instars, but the amount decreased in healthy fruits [16]. [11] Similar pattern in increase of esters can be found in our findings. In addition, some compounds were presented in high concentration in non-infested apple as compare to infested fruit such as propyl isobutyrate, $n$-butyl 2 methylbutyrate and others and also vice versa pattern is seen where high concentration of some compounds can be seen in infested fruits as compare to non infested fruits (Table 2). [11] [17] found that infested mango Mangifera indica (MI) with Anastrepha ludens (Loew) larvae extracts contain many compounds in higher level compared with the other two kinds of mangoes healthy mango (HM) and mechanically damaged mango (MDM). [18] Found nonanol, dodecane, tetradecane, 2-pinene, limonene, farnesene and hexyl carproate in five cultivars of peach infested with Medfly.

\subsubsection{The Volatile Compounds from Orange}

In case of non infested oranges, the main peaks were $\beta$-myrcene, 3 -carene, $\mathrm{D}$ limonene, E-4,8-dimethyl-1,3,7-noatriene, methyl caprylate, isobutyl caproate, ethyl octyate and valencen. Many compounds were detected in fruits infested with third instar larvae but not in fruits infested with first or second instar larvae or in non infested fruit and these included ethyl (Z)-2-butenoate, (+)-2-bornanone, (-)-trans-isopiperitenol, methyl caprate, caryophyllene and farnesene (Table 3). The most significant compounds compared with non infested fruits in third instar were myrcene, 3-carene, D-limonene, methyl caprylate, isobutyl caproate, ethyl octyate and valencen. In the second instar, they were 3-carene, D-limonene, E-4,8-dimethyl-1,3,7-noatriene, methyl caprylate, isobutyl caproate, ethyl octyate and valencen, While for first instar 3-carene, D-limonene, E-4,8dimethyl-1,3,7-noatriene and ethyl octyate were of significant differences compare to non-infested orange (Table 3). Some chemical started to increase in concentration with increase in level of infestation as compare to non infested fruits such as myrcene, D-limonene, $\beta$-cis-ocimene, ethyl octyate and limonene-1,2-dial, while some compounds decreased with level of infestation as compare to non infested, like isobutyl caproate and valencen. In fact, some of these compounds come from peel oil, such as ethyl butanoate, $\beta$-myrcene, and $\alpha$-pinene [19], and others compounds were found in fruit juice including, hexanal, $\beta$-myrcene, cis- $\beta$-ocimene, terpinolene and valencene and other compounds [20]. There were some volatile compounds identified by infested orange with Thaumatotibia leucotreta by using SPME-GC technique, these include $\beta$-myrcene, D-limonene, E-4,8-dimethyl-1,3,7-noatriene, caryophyllene and valencen [21]. D-limonene, n-butyl butyrate, 2-pinene, nonanal, decanal and valencen were detected by infested Grapefruits with immature stages of Caribben fruit fly Anastrepha suspensa (Loew) (Diptera: Tephritidae) [22]. 


\subsubsection{The Volatile Compounds from Mandarin}

Table 3 show the differences between infested and non-infested mandarin fruits. D-limonene was the main peak in both infested and non infested fruit. The fruit infested with third instar of larvae recorded more number of chemicals than other stage of infestation. These new chemicals includes, butanoic acid, 3methyl-,2-methylbutul acetate, sabinene, $\beta$-myrcene, octanoic acid, methyl ester, dihydrocarvone, (-)-trans-isopiperitenol, p-mentha-1(7), 8(10)-dien-9-ol and ethyl laurate which are present only in fruit with third instar larvae (Table 4). Some of these chemicals increase in concentration with increase in infestation like 3-carene, D-limonene, moslene, p-menth-1,4(8)-diene and (+)-epi-bicyclosesquiphellandrene increased from non infested fruit to fruits with third instar larvae. However, tridecane was decreased within first and second instar. From our results, larvae of Medfly have changed the profile of infested fruit especially third instar and this change in profile can be used as an identification tool. Similar results were found by [23] where change in the composition of host fruit odors (volatile profiles) was observed when fruit was infested with medfly and its parasitoid Diachasmimorpha longicaudata (Ashmead). These compounds provide a tool for detection of infestation with fruit fly (Medfly) in the early stage of larvae [21] [14].

\subsubsection{The Volatile Compounds from Lemon}

The highest peaks which were recorded in non infested lemon are sabinene, D-limonene, (E)4,8-dimethyl-1,3,7-noatriene, methyl caprylate, $\alpha$-terpineol, (-)$\beta$-elmene, 7 -epi-a-selinene and intermedol. The results indicated that fruit infested with third instar of larvae recorded a high number of target compounds. These compounds were butyl 2-methylbutanoate, terpinen-4-ol, P-menth-8-en2-one,E-, (3E,7E)-4,8,12-trimethyltrideca-1,3,7,11-tetraene and dodecanoic acid, ethyl ester (Table 5). There were significant differences between infested and non infested lemon fruit in different stages of larvae; these include D-limonene, methyl caprylate, hexyl 2-methylbutyrate and intermedol in third instar. In second instar, D-limonene, methyl caprylate and limonene-1,2-diol. In first instar, they were D-limonene, hexyl 2-methylbutyrate and E-nerolidol. Some of chemicals started to increase in concentration with increase the level of infestation such as sabinene, D-limonene, methyl caprylate and $\alpha$-terpineol, while (-)- $\beta$ elmene decreased within instars. [23] recorded D-limonene, n-butyl butyrate, (-)- $\beta$-elmene and valencen from infested Grapefruits with immature stages of Caribben fruit fly Anastrepha suspensa (Loew) (Diptera: Tephritidae). In addition, [24] found Sabinene, D-limonene, 3-Carene compounds in lemon infested with $D$. citri vector.

\subsubsection{The Volatile Compounds from Avocado}

Sulcatone, D-limonene, E-4,8-dimethyl-1,3,7-noatriene and valencen were the main peaks in avocado fruits. There were some new peaks associated with avocado infestation especially with third instar infestation; these compounds included decane, 3-methyl-, p-menth-1,4(8)-diene, $\alpha$-terpineol, 1,2,6-dimethylun- 
decane and $\alpha$-cubebene. There were significant differences between infested and non infested avocado fruit in different stages of larvae; these include D-limonene, E-4,8-dimethyl-1,3,7-noatriene and valencen for third instar infested fruit. In second instar of larvae, sulcatone, D-limonene, moslene, E-4,8-dimethyl1,3,7-noatriene and valencen. In first instar, they were D-limonene and moslene. Some of chemicals increased in concentration with increase in the level of infestation like D-limonene. Similar results were observed in case of orange, mandarian and lemon. However, 1-heptanal, E-4,8-dimethyl-1,3,7- noatriene and $\alpha$-terpineol decreased in concentration with increase in the level of infestation (Table 6). As mentioned before the number of larvae recorded on avocado fruit was much higher compared to other fruits under laboratory conditions (Figure 1), and this may be due to the volatiles contents which are favourable for the growth of larvae as compare to other types of fruits. Some of the compound detected by [25] includes, hexyl acetate, 2-pinene, valencen and hexyl carproate.

\subsubsection{The Volatile Compounds from Different Fruits Infested with Third Instar Larvae}

In summary, if we compare all five fruits infested with third instar larvae, the major identifying components for each fruits infested with third instar are 1-hexanol, hexyl acetate, n-butyl 2 methylbutyrate and n-hexyl propionate for apple; 3-carene, D-limonene, isobutyl caproate, E-4,8-dimethyl-1,3,7-noatriene and valencen for orange; 3 -Carene and $\mathrm{D}$-limonene for mandarin; Sabinene, $\mathrm{D}$ limonene, (E) 4,8-dimethyl-1,3,7-noatriene, hexyl 2-methylbutyrate, (-)- $\beta$-elmene, 7-epi-a-selinene and e-nerolidol for lemon and finally D-dimonene and valencen were from avocado (Figure 2). D-limonene was present in orange, mandarin, lemon and avocado fruits, but not in apple. Isobutyl caproate were found in orange and avocado fruits. [26] Found that, many volatiles compounds were produced by fruit, which has the same molecular structure with other fruits including, D-limonene and hexyl 2-methylbutyrate

\section{Conclusion}

In conclusion, this paper showed that different types of fruit produce, different volatile organic compound profile as detected by GC-MS and with various larvae instars. Some of these compounds are specifically associated with Medfly infested fruit. In fruits infested with Medfly, the presence of volatile compounds like styrene, decanal in apple, $1-\beta$-pinene, $\beta$-cis-ocimene, isoamyl caproate, limonene-1,2-dial in orange, terebenthene, $\mathrm{p}$-menth-1,4(8)-diene, (-)- $\beta$-elmene, (+)-epi-bicyclosesquiphellandrene in mandarin, sabinene, (E)4,8-dimethyl-1,3, 7-noatriene, $\alpha$-terpineol in lemon and 1,2,6-dimethylundecane, $\alpha$-terpineol in avocado can demonstrate distinction between non-infested and infested fruits. We have shown how Medfly can increase or decrease some of the fruit volatiles. Our results indicate that these volatiles levels, emitted from fruit with an early stage of larvae infestation can be detected by the HS-SPME GC-MS method. Recently, volatiles compound detection technology has been successfully used in different postharvest cases for early infested detection of insects and fungus. 
Fruit infested with Medfly or other insect eggs, release unique volatile compound. These compounds can be exploited to provide tools for improved pest detection. Finally, this research provides the basis for determining the larvae infested by the HS-SPME GC-MS method. Also, it can be used to assess the applicability of this technology for detection of other species of fruit fly, different type of fruits and different number of larvae. We recommend the use this technology in quarantine areas or prior of the importation of fruit for early detection of any infestation in the fruits by fruit flies.

\section{References}

[1] Duyck, P.F. and Quilici, S. (2002) Survival and Development of Different Life Stages of Three Ceratitis spp. (Diptera: Tephritidae) Reared at Five Constant Temperatures. Bulletion of Entomological Research, 92, 461-469.

https://doi.org/10.1079/ber2002188

[2] USDA-APHIS (2006) Exotic Fruit Fly Strategic Plan FY 2006-2010. U.S. Department of Agriculture, Animal and Plant Health Inspection Service. Plant Protection and Quarantine, Oct, 2015.

https://www.aphis.usda.gov/aphis/ourfocus/planthealth/plant-pest-and-disease-pro grams/pests-and-diseases/fruit-flies

[3] NFFS (2010) National Fruit Fly Strategy. Prepared by the NFFS Implementation Committee, April 2010.

http://www.planthealthaustralia.com.au/wp-content/uploads/2014/02/National-Fru it-Fly-Strategy-Implementation-Action-Plan.pdf

[4] Bolognesi, C. and Merlo, F.D. (2011) Pesticides: Human health Effects. In: Journal of Nrjagu, Ed., Encyclopedia of Environmental Health, Elsevier, Burlington, 438453.

[5] Wang, Y., Yang, C., Li, S., Yang, L., Wang, Y. and Zhao, J. (2009) Volatile Characteristics of 50 Peaches and Nectarines Evaluated by HS-SPME with GC-MS. Food Chemistry, 116, 356-364.

[6] Horvat, R.J., Chapman, G.W., Robertson, J.A., Meredith, F.I., Scorza, R. and Callahan, A.M. (1990) Comparison of the Volatile Compounds from Several Peach Cultivars. Journal of Agricultural and Food Chemistry, 36, 234-237. https://doi.org/10.1021/jf00091a051

[7] Thomas, D.B., Epsky, N.D., Serra, C.A., Hall, D.G., Kendra, P.E. and Heath, R.R. (2008) Ammonia Formulations and Capture of Anastrepha Fruit Flies (Diptera: Tephritidae). Journal of Entomology Science, 43, 76-85.

[8] Gould, W.P. (1995) Probability of Detecting Caribbean Fruit Fly (Diptera: Tephritidae) by Fruit Dissection. Florida Entomologist, 78, 502-507. https://doi.org/10.2307/3495535

[9] USDA-APHIS (2010) Fresh Fruits and Vegetables Import Manual. U.S. Department of Agriculture, Animal and Plant Health Inspection Service. Plant Protection and Quarantine.

http://www.aphis.usda.gov/importexport/plants/manuals/ports/downloads/fv.pdf

[10] Howe, G.A. and Jander, G. (2008) Plant Immunity to Insect Herbivores. Annu. Rev. Plant Biol., 59, 41-66. https://doi.org/10.1146/annurev.arplant.59.032607.092825

[11] Carrasco, M., Montoya, P., Cruz-Lopez, L. and Rojas, J.C. (2005) Response of the Fruit FLY parasitoid Diachasmimorpha longicaudata (Hymenoptera: Braconidae) to Mango Fruit Volatiles. Environmental Entomology, 34, 576-583. 
https://doi.org/10.1603/0046-225X-34.3.576

[12] Neto, A.M., Tatiana, R.O., Vanessa. S., Benevides, C., Mariangela, V.L, Deise, C., Jair, V., Givanildo, B.O., Julio, M.M., Beatriz, A.J. and Antônio, N. (2012) Mass-Rearing of Mediterranean Fruit Fly Using Low-Cost Yeast Products Produced in Brazil. Sci. Agric., 69, 364-369. https://doi.org/10.1590/S0103-90162012000600004

[13] SAS University Edition (2012) SAS Guide for Personal Computers, Version 8.2. SAS University Edition.

[14] Chamberlain, K., Mathilde, B., Jennifer, H.J., Suzanne, J.C. and John, A.P. (2012) Use of Honey Bees (Apis mellifera L.) to Detect the Presence of Mediterranean Fruit Fly (C.capitata Wiedemann) Larvae in Valencia Oranges. J. Sci. Food Agric., 92, 2050-2054. https://doi.org/10.1002/jsfa.5742

[15] Hernandez, M.M., Avispuro, I.V., Sanz, I., Adelantado, M. and Yafera, E.P. (1999) Electroantennogram Activity Assay of $C$. capitata to Airborne Volatiles from Peach of Three Ripeness Stages. J. of South-western Entomologist, 24, 133-142.

[16] Hern, A. and Dorn, S. (2001) Induced Emissions of Apple Fruit Volatiles by the Codling Moth: Changing Patterns with Different Time Periods after Infestation and Different Larval Instars. Phytochemistry, 57, 409-416.

[17] Li, Z., Ning, W., Raghavan, G.S. and Vigneault, C. (2009) Ripeness and Rot Evaluation of 'Tommy Atkins' Mango Fruit through Volatiles Detection. Journal of Food Engineering, 91, 319-324.

[18] Tabilio , M.R., Dennis, F., Enrico, M., Stefano, M., Maurizio, D.R. and Salvador, S.M. (2013) Impact of the Mediterranean Fruit Fly (Medfly) C. capitata on Different Peach Cultivars: The Possible Role of Volatile Peach Compounds. Food Chemistry, 140, 375-381.

[19] Bylaite, E. and Anne, S.M. (2005) Characterisation of Volatile Aroma Compounds of Orange Juices by Three Dynamic and Static Headspace Gas Chromatography Techniques. European Food Research and Technology, 222, 176. https://doi.org/10.1007/s00217-005-0141-8

[20] Qiao, Y., Bi, J.X., Yan, Z., Yun, Z., Gang, F., Xiao, L.Y. and Si, Y.P (2008) Characterization of Aroma Active Compounds in Fruit Juice and Peel Oil of Jinchen Sweet Orange Fruit (Citrus sinensis (L.) Osbeck) by GC-MS and GC-O. Molecules, 13, 1333-1344. https://doi.org/10.3390/molecules13061333

[21] Zimba, K., Martin, P.H., Sean, D.M. and Unathi, H. (2015) Agathis bishopi (Hymenoptera: Braconidae) as a Potential Tool for Detecting Oranges Infested with Thaumatotibia leucotreta (Lepidoptera: Tortricidae). Journal of Insect Behaviour, 28, 618-633. https://doi.org/10.1007/s10905-015-9526-0

[22] Kendra, P.E., Amy, L.R., Wayne, S.M., Elena, Q.S., Jerome, N., Nancy, D.E. and Robert, R.H. (2011) Gas Chromatography for Detection of Citrus Infestation by Fruit Fly Larvae (Diptera: Tephritidae). Postharvest Biology and Technology, 59, 143-149.

[23] Ovruski, S.M., Bendjian, L.R., Van, G.A., Medina, N. and Schliserman, P. (2011) Host Preference by D. longicaudala Reared on Larvae of A. fraterculus and C. capitata. Florida Entomologist, 94, 195-200. https://doi.org/10.1653/024.094.0211

[24] Mann, R.S., Jared, G.A., Sara, L.H., Siddharth, T., Kirsten, S.P., Hans, T.A. and Lukasz, L.S. (2012) Induced Release of a Plant-Defense Volatile "Deceptively" Attracts Insect Vectors to Plants Infected with a Bacterial Pathogen. PLoS Pathogens, 3, 1-13. https://doi.org/10.1371/journal.ppat.1002610

[25] Mercedes, G.L., Guzmánb, G.R. and Dorantes A.L. (2004) Solid-Phase Microextrac- 
tion and Gas Chromatography-Mass Spectrometry of Volatile Compounds from Avocado Puree after Microwave Processing. Journal of Chromatography, 1036, 8790.

[26] Jang, E.B., Light, D.M., Flath, R.A., Nagata, J.T. and Mon, T.R. (1989) Electroantennogram Responses of the Mediterranean Fruit Fly, Ceratitis capitata to Identified Volatile Constituents from Calling Males. Entomologia Experimentalis et Applicata, 50, 7-19. https://doi.org/10.1111/j.1570-7458.1989.tb02307.x

Submit or recommend next manuscript to SCIRP and we will provide best service for you:

Accepting pre-submission inquiries through Email, Facebook, LinkedIn, Twitter, etc. A wide selection of journals (inclusive of 9 subjects, more than 200 journals) Providing 24-hour high-quality service User-friendly online submission system Fair and swift peer-review system Efficient typesetting and proofreading procedure Display of the result of downloads and visits, as well as the number of cited articles Maximum dissemination of your research work

Submit your manuscript at: http://papersubmission.scirp.org/ Or contact jbm@scirp.org 